\title{
Pathogenesis of hemangioma
}

\author{
Douglas A. Marchuk
}

Commentary

See related article, pages $745-752$.

Department of Genetics, Box 3175, Duke University Medical Center, Durham,

North Carolina 27710, USA. Phone: (919) 684-3290; Fax: (919) 681-9193; E-mail: march004@mc.duke.edu.

Hemangioma is the most common tumor of any kind seen in infancy. It is also, perhaps, the least understood. This stems in part from a long history of confusing nomenclature for vascular anomalies that employed classification schemes based on superficial descriptions of the lesions. The first major step toward clarifying this nomenclatural morass was made by Mulliken and colleagues $(1,2)$, who employed a biological classification scheme based on the differing clinical courses and endothelial proliferative activity of hemangiomas versus malformations. Hemangiomas, often called infantile or juvenile hemangiomas for clarity, are benign tumors that exhibit an early and rapid proliferation phase during the first year of life characterized by endothelial and pericytic hyperplasia, followed by a slower but steady involution phase that may last for years. This basic scheme of histopathological classification has been refined by immunohistochemical characterization of hemangioma in each of the phases (3). Nonetheless, the pathogenesis of hemangioma is still not understood. Although growth factors and hormonal and mechanical influences have been postulated to affect the abnormal proliferation of endothelial cells in hemangioma, the primary, causative defect in hemangiogenesis remains unknown and no genetic alteration has been implicated.

This dearth of molecular details is striking, considering the growing list of germline mutations in genes causing specific inherited syndromes involving vascular malformations, including hereditary hemorrhagic telangiectasia $(4,5)$, cutaneous venous malformations (6), cerebral cavernous malformations $(7,8)$, and hyperkeratotic cutaneous capillary-venous malformation (9). Are we able to investigate hemangioma in a similar manner? Cheung and colleagues (10) compared the concordance of hemangioma in monozygotic versus dizygotic twins and found no evidence of a strong predisposing inherited component. However, Blei and colleagues described six rare families segregating hemangiomas and/or vascular malformations as an autosomal dominant trait with incomplete penetrance (11). This suggested a predisposing mutation in these families segregating the trait. Genetic involvement was bolstered with the genetic mapping of a locus on chromosome $5 q$ for hemangioma/malformation development in these particular families (12), but the gene responsible has yet to be identified. In addition, it is not certain that this gene plays any role in the more common sporadic hemangiomas.

Into this gene-deficient story comes the paper of Boye and colleagues (13) in this issue of the JCI, showing that endothelial cells derived from hemangioma are clonal in origin and demonstrating that they arise from a common precursor cell. Significantly, fibroblastlike cells isolated from these tumors are not clonal, as determined using a wellestablished assay based on X-chromosome inactivation in females.

\section{Cell-autonomous defects in hemangiomas}

The data of Boye et al. (13) support the hypothesis that the primary defect is intrinsic to endothelial cells, rather than other cell types constituting the hemangioma. A competing hypothesis, holding that proliferation of endothelial cells is primarily a response to factors secreted by neighboring cells, can therefore be ruled out. Although stromal cells (14) and the overlying epidermis of hemangiomas (15) exhibit altered properties, including aberrant expression of angiogenic factors, these endothelial cells would not be expected to develop as clones of a single founder cell if they were merely responding to an externally derived angiogenic signal.

The clonality data are intriguing in one other aspect as well. When Williams et al. (16) injected mouse endothelioma cells expressing the polyoma middle- $T$ oncogene into adult mice, they observed that the resulting tumors resemble benign hemangiomas, but they showed that the endothelial cells within these tumors are primarily hostderived, apparently recruited by the injected oncogenic cells. The results obtained by Boye et al. for hemangioma in infants (13) show no evidence of additional endothelial recruitment by the original endothelial precursor cells of the tumor. If additional endothelial cells are indeed recruited into the tumor, it appears that they retain their nonproliferative phenotype. It would be interesting to determine whether the endothelial cells directly obtained from the tumor, prior to selection via growth in vitro, also retain their clonality.

Boye and colleagues (13) show that the endothelial cells they isolated from hemangiomas exhibit enhanced proliferation and migration, in keeping with the rapid growth of the vascular lesion in the neonate. However, there was one surprise: In the presence of the angiogenic inhibitor endostatin, migration of these cells was not inhibited but rather stimulated, suggesting a radically altered cellular phenotype. One possible explanation, favored by the authors, is that a precursor endothelial cell had undergone a mutation in a gene regulating angiogenesis, resulting in clonal expansion. Evidence for somatic mutations in hemangioma comes from Berg and colleagues, who showed that loss of heterozygosity $(\mathrm{LOH})$ in hemangioma tissue is prevalent on chromosome 5q (17), suggesting that loss-of-function mutations contributes to hemangioma development. Research over the past decade has shown that angiogenesis is tightly regulated by a balance of promoting and inhibitory factors. One way to shift the balance toward aberrant proliferation would be to inactivate one of these inhibitory factors. Thus, the LOH on chromosome $5 \mathrm{q}$ suggests that an inhibitory factor maps to this chromosomal region, which, when mutated, promotes hemangioma development.

An equally likely possibility is a somatic mutation leading to constitutive activation of an angiogenesis-promoting 
gene. A number of candidate genes come to mind, some of which are suggested by Boye et al. (13). These genes can be sequenced in the isolated endothelial cells to identify somatic mutations responsible for hemangiogenesis.

\section{Developmental origins of hemangiomas}

An alternative interpretation of the present data comes from work of North and colleagues, who have documented the expression of placental vascular epitopes in hemangiomas. Hemangiomas display high levels of immunostaining for the GLUT1 glucose transporter (18), a surface protein that is highly expressed in most embryonic and fetal endothelial cells but is lost in most tissues except at the blood-tissue barriers, including microvessels in the central nervous system and the placenta. North et al. have expanded on this initial finding to show that other antigens associated with placental vessels, including FcyRII, Lewis Y antigen, and merosin, are also expressed in hemangioma (19).

The similarities in gene expression between hemangiomal and placental vessels might be explained by a somatic mutation in a regulatory gene that directs hemangiomal endothelial cells toward a placental phenotype, but North and colleagues also suggest an alternative theory (19). Embolic placental endothelial cells could reach fetal tissues from chorionic villi through right-to-left shunts characteristic of the normal fetal circulation. If the embolus contained a single endothelial cell or only a small number of endothelial cells, this would also be compatible with the clonality results obtained by Boye and colleagues (13). Because the placental endothelial cells involved might well be of fetal rather than maternal origin, these models are not easily distinguished, short of identifying somatic mutations in genes that can be subsequently shown to induce hemangioma formation. Nevertheless, the placental origin theory is attractive, because it would explain the exclusively perinatal or congenital presentation of hemangiomas. The otherwise puzzling observation that chorionic villus sampling increases the risk of hemangiomas $(20,21)$ further supports this model, since the local placental injury caused in this procedure might increase shedding of endothelial cells from chorionic villi into the fetal circulation.

Hemangiomas pose other perplexing questions that will only be answered as the events that initiate hemangiogenesis are elucidated. For example, the strong gender predilection of hemangioma toward female over male infants (3:1 or more) suggests hormonal effects in hemangiogenesis. In addition, the anatomical predilection for the head and neck region of juvenile hemangioma must be explained. Perhaps most intriguing from a therapeutic standpoint is the spontaneous involution of the lesion. This distinguishing characteristic has been shown to be due in part to apoptosis of the endothelial cells (22), but the trigger for this process remains unknown. Can this apoptotic program be switched on earlier and be accelerated?

Whatever mechanisms are identified in hemangiogenesis, we have come a long way from the descriptive phase of hemangioma research. The intriguing data of Boye and colleagues (13) suggest one mechanism for hemangioma formation and bring the field a step closer to understanding the molecular etiology of this common tumor.

1. Mulliken, J., and Glowacki, J. 1982. Hemangiomas and vascular malformations in infants and children: a classification based on endothelial characteristics. Plast. Reconstr. Surg. 69:412-420.

2. Mulliken, J., and Young, A.E. 1988. Vascular birthmarks: hemangiomas and vascular malformations. W.B. Saunders Co. Philadelphia, Pennsylvania, USA. 24-37.

3. Takahashi, K., et al. 1994. Cellular markers that distinguish the phases of hemangioma during infancy and childhood. J. Clin. Invest. 93:2357-2364.

4. McAllister, K.A., et al. 1994. Endoglin, a TGF-beta binding protein of endothelial cells, is the gene for hereditary haemorrhagic telangiectasia type 1. Nat. Genet. 8:345-351.

5. Johnson, D.W., et al. 1996. Mutations in the activin receptor-like kinase 1 gene in hereditary hemorrhagic telangiectasia type 2. Nat. Genet. 13:189-195.

6. Vikkula, M., et al. 1996. Vascular dysmorphogenesis caused by an activating mutation in the receptor tyrosine kinase TIE-2. Cell. 87:1181-1190.

7. Laberge-le Couteulx, S., et al. 1999. Truncating mutations in CCM1, encoding KRIT1, cause hereditary cavernous angiomas. Nat. Genet. 23:189-193.

8. Sahoo, T., et al. 1999. KRIT1, a Krev1/rapla binding protein, is mutated in cerebral cavernous malformations (CCM1). Hum. Mol. Genet. 8:2325-2334.

9. Eerola, I., et al. 2000. KRIT1 is mutated in hyperkeratotic cutaneous capillary-venous malformation associated with cerebral capillary malformation. Hum. Mol. Genet. 9:1351-1355.

10. Cheung, D.S., Warman, M.L., and Mulliken, J.B. 1997. Hemangioma in twins. Ann. Plast. Surg. 38:269-274.

11. Blei, F., Walter, J., Orlow, S.J., and Marchuk, D.A. 1998. Familial segregation of hemangiomas and vascular malformations as an autosomal dominant trait: a rare genetic disorder. Arch. Dermatol. 134:718-722.

12. Walter, J.W., et al. 1999. Genetic mapping of a novel familial form of infantile hemangioma. Am. J. Med. Genet. 82:77-83.

13. Boye, E., et al. 2001. Clonality and altered behavior of endothelial cells from hemangiomas. J. Clin. Invest. 107:745-752.

14. Berard, M., et al. 1997. Vascular endothelial growth factor confers a growth advantage in vitro and in vivo to stromal cells cultured from neonatal hemangiomas. Am. J. Pathol. 150:1315-1326.

15. Bielenberg, D.R., et al. 1999. Progressive growth of infantile cutaneous hemangiomas is directly correlated with hyperplasia and angiogenesis of adjacent epidermis and inversely correlated with expression of the endogenous angiogenesis inhibitor, IFN-beta. Int. J. Oncol. 14:401-408.

16. Williams, R.L., et al. 1989. Endothelioma cells expressing the polyoma middle $\mathrm{T}$ oncogene induce hemangiomas by host cell recruitment. Cell. 57:1053-1063.

17. Berg, J.N., et al. 2001. Evidence for loss of heterozygosity of $5 \mathrm{q}$ in sporadic haemangiomas: are somatic mutations involved in haemangioma formation? J. Clin. Pathol. In press.

18. North, P.E., Waner, M., Mizeracki, A., and Mihm, M.C., Jr. 2000. GLUT1, a newly discovered immunohistochemical marker for juvenile hemangiomas. Hum. Pathol. 31:11-22.

19. North, P.E., et al. 2001. A unique microvascular phenotype shared by juvenile hemangiomas and human placenta. Arch. Dermatol. In press.

20. Kaplan, P., et al. 1990. Malformations and minor anomalies in children whose mothers had prenatal diagnosis: comparison between CVS and amniocentesis. Am. J. Med. Genet. 37:366-370.

21. Burton, B.K., Schulz, C.J., Angle, B., and Burd, L.I. 1995. An increased incidence of haemangiomas in infants born following chorionic villus sampling (CVS). Prenat. Diagn. 15:209-214.

22. Razon, M.J., Kraling, B.M., Mulliken, J.B., and Bischoff, J. 1998. Increased apoptosis coincides with onset of involution in infantile hemangioma. Microcirculation. 5:189-195. 